\title{
Impact of Patient- and Clinician-Reported Cumulative Toxicity on Quality of Life in Patients With Metastatic Castration-Naïve Prostate Cancer
}

\author{
Claudia S.E.W. Schuurhuizen, MDª, ; Patricia Marino, $\mathrm{PhD}^{\mathrm{c}}$; Annemarie M.J. Braamse, $\mathrm{PhD}^{\mathrm{d}}$; \\ Laurien M. Buffart, PhDa,e; Florence Joly, MD, PhD; ; Karim Fizazi, MD, PhDs; Muriel Habibian, $\mathrm{PhD}^{\text {h }}$; \\ Jean-Marie Boher, $\mathrm{PhD}^{\mathrm{i}, j}$; Michel Soulie, MD, $\mathrm{PhD}^{\mathrm{k}}$; Stéphane Oudard, MD, PhD; \\ Inge R.H.M. Konings, MD, PhDa; Henk M.W. Verheul, MD, PhDª Joost Dekker, $\mathrm{PhD}^{\mathrm{b}}$; \\ and Gwenaelle Gravis, MD ${ }^{\mathrm{m}}$
}

\begin{abstract}
Background: Current toxicity evaluation is primarily focused on high-grade adverse events (AEs) reported by clinicians. However, the cumulative effect of multiple lower-grade AEs may also impact patients' quality of life (QoL). Further, patient-reported toxicity may be more representative of patients' treatment experiences. This study aimed to determine whether cumulative toxicity comprising all-grade AEs is more associated with QoL than cumulative toxicity comprising high-grade AEs only, and whether patient-reported cumulative toxicity is more associated with QoL than clinician-reported cumulative toxicity. Methods: Patients with metastatic castration-naïve prostate cancer participating in the phase III GETUG-AFU 15 trial completed questionnaires on AEs (at 3 and 6 months) and QoL (at baseline and 3 and 6 months). Clinicians reported AEs during clinical visits. Cumulative toxicity scores were calculated for clinicians and patients in 3 ways: total number of high-grade AEs, total number of all-grade AEs, and total number of all AEs multiplied by their grade (severity score). Relationships between cumulative toxicity scores and QoL were studied using longitudinal regression analyses; unstandardized (B) and standardized regression coefficients $(\beta)$ are reported. Results: Of 385 patients, 184 with complete QoL and toxicity data were included. Clinician-reported all-grade $A E s(B,-2.2 ; 95 \% \mathrm{Cl},-3.3$ to $-1.1 ; P<.01)$ and severity score $(B,-1.4 ; 95 \% \mathrm{Cl},-2.2$ to $-0.7 ; P<.01)$ were associated with deteriorated physical QoL, whereas the total number of high-grade AEs was not. All patient-reported scores were significantly $(P<.01$ for all) associated with deteriorated physical and global QoL. Standardized regression coefficients indicated that patient-reported toxicity scores were more associated with QoL outcomes than clinician-reported scores, with the strongest association found for the all-grade AEs and severity cumulative toxicity scores. Conclusions: Patient- and clinician-based cumulative toxicity scores comprising all-grade AEs better reflect impact on patient QoL than toxicity scores comprising high-grade AEs only. To assess the effect of toxicity on QoL, patient-reported cumulative toxicity scores are preferred.
\end{abstract}

Cancerologie de Marseille (CRCM), Marseille, France.

Submitted February 21, 2018; accepted for publication August 3, 2018. Dr. Verheul has disclosed that he has received grant/research support from Roche, Vitromics Healthcare System, Novartis, Immunovo B.V., and Amgen; nonfinancial support from Pfizer; and honoraria from Boehringer Ingelheim. Dr. Joly has disclosed that she has received grant/research support from Sanofi, and that she has served on the advisory board for Sanofi and Janssen. The remaining authors have disclosed that they have no financial interests, arrangements, affiliations, or commercial interests with the manufacturers of any products discussed in this article or their competitors.

Drs. Dekker and Verheul received a grant from Alpe d'Huzes/Dutch Cancer Society (VU 2011-5279) during the conduct of the study.

Correspondence: Henk M.W. Verheul, MD, PhD, Department of Medical Oncology, VU University Medical Center, Cancer Center Amsterdam, P.O. Box 7057, 1081 HV, Amsterdam, the Netherlands.

Email: h.verheul@vumc.nl 
In daily oncology practice, clinicians constantly weigh the expected benefit of treatment against exposure to possible treatment-related adverse events (AEs). ${ }^{1}$ Especially in patients with advanced disease, for whom the survival benefit of (systemic) treatments may be limited, the number and extent of AEs and their impact on patient quality of life (QoL) are important. ${ }^{2}$ Providing a representative overview of treatment-related AEs is essential for patients to make an informed decision to undergo treatment. ${ }^{3,4}$

Currently, the main source of information on toxicity in randomized controlled trials (RCTs) is a clinician-based assessment of AEs during clinic visits using the NCI Common Terminology Criteria for Adverse Events (CTCAE). ${ }^{5,6}$ However, this may be suboptimal, because the reporting of AEs in RCTs is primarily focused on high-grade AEs (grades 3-4), whereas the lower-grade AEs are not always incorporated. ${ }^{7}$ Although a description of high-grade toxicities is important and relevant to determine drug safety, patient QoL is also likely to be influenced by the often daily (re)occurring, longer-lasting grade 1 or 2 AEs throughout therapy, because these determine drug tolerability. We recently studied the burden of cumulative toxicity in patients with colorectal cancer (CRC) and found that cumulative toxicity scores comprising all-grade AEs were associated with patients' physical QoL, whereas the total score of high-grade AEs was not. ${ }^{8}$ The cumulative toxicity scores reported in our study were based on clinicianreported AEs, whereas the accuracy of assessment and reporting of AEs by clinicians has been recently questioned. ${ }^{8}$ Symptomatic AEs associated with anticancer treatments seem to be underreported by clinicians and when data are prospectively collected within RCTs. ${ }^{9}{ }^{910}$ Patient-reported outcomes to assess AEs may provide an alternative measure for patients' treatment experiences. ${ }^{11}$

The purpose of this prospective study in patients with metastatic castration-naïve prostate cancer (mCNPC) was to determine whether cumulative toxicity comprising all-grade AEs (grades 1-4) is more associated with QoL than cumulative toxicity comprising high-grade AEs only (grades 3-4), and whether cumulative toxicity reported by patients is more associated with QoL than cumulative toxicity reported by clinicians.

\section{Methods}

This is a secondary analysis of data obtained in the prospective GETUG-AFU 15 trial, ${ }^{12}$ which was a multicenter, randomized, open-label phase III trial that evaluated the efficacy and safety of docetaxel combined with androgen deprivation therapy (ADT) compared with ADT alone on overall survival in patients with mCNPC. The study was approved by the French Comités de Protection des Personnes and written consent was obtained for all patients (ClinicalTrials.gov identifier: NCT00104715). Results of the original trial have been published previously. ${ }^{13}$ In brief, no significant difference in overall survival was seen between the treatment arms, although progression-free survival was significantly improved in the docetaxel group.

\section{Patients}

A total of 385 patients with mCNPC were enrolled between October 2004 and December 2008 in the GETUG-AFU 15 trial. For the current analyses, we included patients who completed QoL assessments before and 3 or 6 months after the start of treatment, and for whom patient- and clinician-reported toxicity data were available.

\section{Study Design and Measurements}

Patients completed QoL questionnaires at baseline (before the start of first-line treatment) and after 3 and 6 months of treatment. Clinicians reported on AEs during clinical visits, which occurred every 3 weeks in the ADT + docetaxel group and every 3 months in the ADT-alone group. Patients were invited to complete a questionnaire on AEs 3 and 6 months after the start of treatment, immediately before or after toxicity evaluation by their clinicians. This questionnaire was adapted from a previous study ${ }^{14}$ and consisted of 26 items describing 22 symptoms often associated with docetaxel and castration treatments. For each symptom, patients were asked whether it occurred during the previous month (yes/no) and the extent of subjective disturbance that they experienced (using a 4-point Likert scale ranging from $1=$ not at all; $4=$ very much).

We looked for the same 22 symptoms reported by clinicians at the 3-and 6-month clinical visit from patient records and graded these using NCICTCAE, version 3.0. 
CTCAE items representing single AEs were graded on a 4 -point Likert scale $(1=$ mild; $4=$ lifethreatening). Grade 3 and 4 AEs generally indicate the need for clinical action. ${ }^{6}$ If a symptom was not mentioned, it was considered absent. Following the approach from our previous study, ${ }^{8}$ physical function and global QoL were assessed with subscales of the EORTC Quality of Life Questionnaire-Core 30 (EORTC QLQ-C30); this cancer-specific QoL questionnaire is internationally validated and widely used. ${ }^{13}$ A change in QoL of at least 10 points is regarded as a minimal clinically important difference. ${ }^{15,16}$ Additionally, patient demographic, tumor, and treatment characteristics and ECOG performance status (PS) were extracted from patient records. Progression during the first 3 or 6 months of treatment comprised biochemical progression, clinical progression, or death as defined in the original trial. $^{12}$

\section{Statistical Analysis}

Descriptive analyses were performed to summarize patient demographic, tumor, and treatment characteristics and QoL. Multivariate logistic regression analyses were used to compare characteristics of patients from the GETUG-AFU 15 trial who were included in the current analyses versus those who were excluded, and to compare characteristics of patients with missing data versus those with intact data. Prevalence of the specific types of CTCAE grade AEs was calculated. For each patient, we calculated the clinician-based cumulative toxicity scores in 3 ways: (1) high-grade cumulative toxicity score as the total number of severe AEs (grades 3-4), (2) allgrade cumulative toxicity score as the total number of AEs (all grades), and (3) cumulative toxicity severity score as the sum of the total number of all AEs multiplied by their grade. ${ }^{8}$

Each of the 22 symptoms reported as being present by patients was graded from 1 to 4 , analogous to the rating of subjective disturbance. When symptoms were absent, they were coded as 0 . Subsequently, patient-reported cumulative toxicity scores were calculated in an identical manner as the clinicianbased cumulative toxicity scores. To test the degree of agreement between the mean cumulative toxicity scores reported by patients and clinicians, intraclass correlation coefficients (ICCs) were calculated using two-way random-effects models. ${ }^{17}$ ICC values $<0.5$ indicate poor agreement, between 0.5 and 0.75 are moderate, between 0.75 and 0.9 are good, and $>0.9$ are excellent. ${ }^{17}$ Mean changes in physical and global QoL over time were evaluated using longitudinal regression analyses.

Associations between each of the cumulative toxicity scores and physical and global QoL at 3 and 6 months were evaluated using longitudinal regression analyses (linear mixed models [LMM]). The models were adjusted for QoL at baseline. ${ }^{18}$ The LMM handles missing data automatically under the missingat-random assumption. We built separate models for each of the clinician- and patient-reported cumulative toxicity scores. In the multivariate regression models, we adjusted for the following covariates: age, ECOG PS, progression during treatment (yes/ no), baseline QoL, allocation to treatment arm in the GETUG-AFU 15 trial, metastatic volume (high vs low, with high metastatic volume defined as the presence of visceral metastases and/or at least 4 bone lesions, including at least 1 bone structure beyond the spine or pelvis), ${ }^{19}$ serum concentration of prostate-specific antigen at treatment initiation $(<65$ or $\geq 65 \mathrm{ng} / \mathrm{mL}$ ), and Gleason score at baseline (2-6 vs 7 vs $8-10)$.

Unstandardized (B) and standardized regression coefficients $(\beta)$ and 95\% CIs were reported, allowing clinical interpretation (B) and direct comparison $(\beta)$ of the obtained regression coefficients in the different models, respectively. The standardized regression coefficients were determined by performing the LMM on the QoL and toxicity data after converting these data into $\mathrm{Z}$ scores. ${ }^{20}$ For all statistical analyses, $P<.05$ was considered statistically significant. Data were analyzed using SPSS Statistics, version 22 (SPSS Inc.).

\section{Results}

Patient characteristics of the GETUG-AFU 15 trial are presented in Table 1 . Of the 385 patients enrolled, 201 were not eligible for current analyses due to completely missing QoL questionnaires and/ or toxicity data (Figure 1). Consequently, data from 184 patients were included. No significant differences in baseline demographic and clinical characteristics were seen between the 184 patients included and the 201 patients excluded from the analyses (Table 1). Cumulative clinician-reported scores were avail- 
Schuurhuizen et al

\begin{tabular}{|c|c|c|c|c|}
\hline & $\begin{array}{l}\text { GETUG-AFU } 15 \\
(\mathrm{~N}=385)\end{array}$ & $\begin{array}{l}\text { Excluded From } \\
\text { Cumulative AE Study } \\
(\mathrm{N}=201)\end{array}$ & $\begin{array}{c}\text { Included in } \\
\text { Cumulative AE Study } \\
(\mathrm{N}=184)\end{array}$ & $P$ Value \\
\hline Age, y & & & & .183 \\
\hline Mean (SD) & $63(7.8)$ & $62.6(7.8)$ & $63.5(7.8)$ & \\
\hline Range & $43-84$ & $43-81$ & $48-84$ & \\
\hline Serum concentration of prostate-specific antigen, $\mathrm{ng} / \mathrm{mL}$ & & & & .924 \\
\hline Mean (SD) & $197.3(808.6)$ & $271.7(1,084.6)$ & $116.9(281.7)$ & \\
\hline Range & $0-11,900$ & $0-11,900$ & $0-2,780$ & \\
\hline Missing & 4 & 3 & 1 & \\
\hline Metastatic extent, n (\%) & & & & .744 \\
\hline High-volume disease & $183(47.2 \%)$ & $96(47.8 \%)$ & $87(47.3 \%)$ & \\
\hline Low-volume disease & $202(52.5 \%)$ & $105(52.2 \%)$ & $97(52.7 \%)$ & \\
\hline Treatment arm, n (\%) & & & & .077 \\
\hline ADT + docetaxel & $191(49.6 \%)$ & $86(42.8 \%)$ & $105(57.1 \%)$ & \\
\hline ADT & $194(50.4 \%)$ & $115(57.2 \%)$ & $79(42.9 \%)$ & \\
\hline Initial Gleason score, n (\%) & & & & .124 \\
\hline $2-6$ & $32(8.3 \%)$ & $13(6.5 \%)$ & $19(10.3 \%)$ & \\
\hline 7 & $130(33.8 \%)$ & $63(31.3 \%)$ & $67(36.4 \%)$ & \\
\hline $8-10$ & $216(56.1 \%)$ & $122(60.7 \%)$ & $94(51.1 \%)$ & \\
\hline Missing & $7(1.8 \%)$ & $3(1.5 \%)$ & $4(2.2 \%)$ & \\
\hline ECOG performance score, $n(\%)$ & & & & .493 \\
\hline 0 & $357(97.7 \%)$ & $182(90.5 \%)$ & $175(95.1 \%)$ & \\
\hline 1 & $9(2.3 \%)$ & $6(3.0 \%)$ & $3(1.6 \%)$ & \\
\hline Missing & $19(4.9 \%)$ & $13(6.5 \%)$ & $6(3.3 \%)$ & \\
\hline EORTC QLQ-C30 global QoL score & & & & .648 \\
\hline Mean (SD) & $66.6(21.1)$ & $66.2(20.7)$ & $66.9(21.3)$ & \\
\hline Range & $0-100$ & $0-100$ & $0-100$ & \\
\hline Missing & 86 & 86 & 0 & \\
\hline EORTC QLQ-C30 physical functioning score & & & & .450 \\
\hline Mean (SD) & $87.8(16.8)$ & $88.7(21.3)$ & $87.2(16.6)$ & \\
\hline Range & $20-100$ & $20-100$ & $20-100$ & \\
\hline Missing & 78 & 78 & 0 & \\
\hline
\end{tabular}

Abbreviations: ADT, androgen deprivation therapy; AE, adverse event; QLQ-C30, Quality of Life Questionnaire Core-30; QoL, quality of life.

able for $183(99.5 \%)$ and $120(65.2 \%)$ patients at 3 and 6 months after treatment initiation, respectively (Table 2). Additionally, 168 patients (91.3\%) completed the patient-reported AEs at 3 months after start of treatment, and 107 patients $(58.2 \%)$ at 6 months after start of treatment (Table 2). Data were missing for $\geq 1$ QoL or toxicity assessment for 100 patients. Patients with missing values were more likely to have been allocated to the intervention (ADT + docetaxel) arm (68\% vs 44\%) and to have had progressive disease (12\% vs $7 \%$ ) during treatment than patients without missing values (data not shown).

Mean age (SD) of patients included in the current study was 63.5 (7.8) years, and $95.1 \%$ of patients had an ECOG PS of 0 before start of treatment. Mean QLQ-C30 global QoL score changed significantly over time, from 66.9 (21.3) at baseline to $68.0(19.3)$ at 3 months and $64.4(19.4)$ at 6 months after start of treatment $(P=.041)$. Mean QLQ-C30 physical functioning score reduced significantly over time, from $87.2(16.6)$ at baseline to $83.4(18.0)$ at 3 months and $79.3(18.8)$ at 6 months after start of treatment $(P \leq .001)$.

\section{Reporting of AEs and Cumulative Toxicity Scores}

Clinicians reported AEs for 159 (86.9\%) and 108 (90\%) patients between the first 3 months and between 3 and 6 months after start of treatment, respectively. At least one high-grade AE (grade 3-4) was reported in 19 patients $(10.4 \%)$ during the first 3 months of treatment, and in $24(20 \%)$ between 3 and 6 months (Table 2). All patients reported AEs between the first 3 months and between 3 and 6 months after start of treatment. In total, at least one high-grade AE (grade 3-4) was reported by 149 patients $(88.7 \%)$ after 3 months of treatment and by 93 


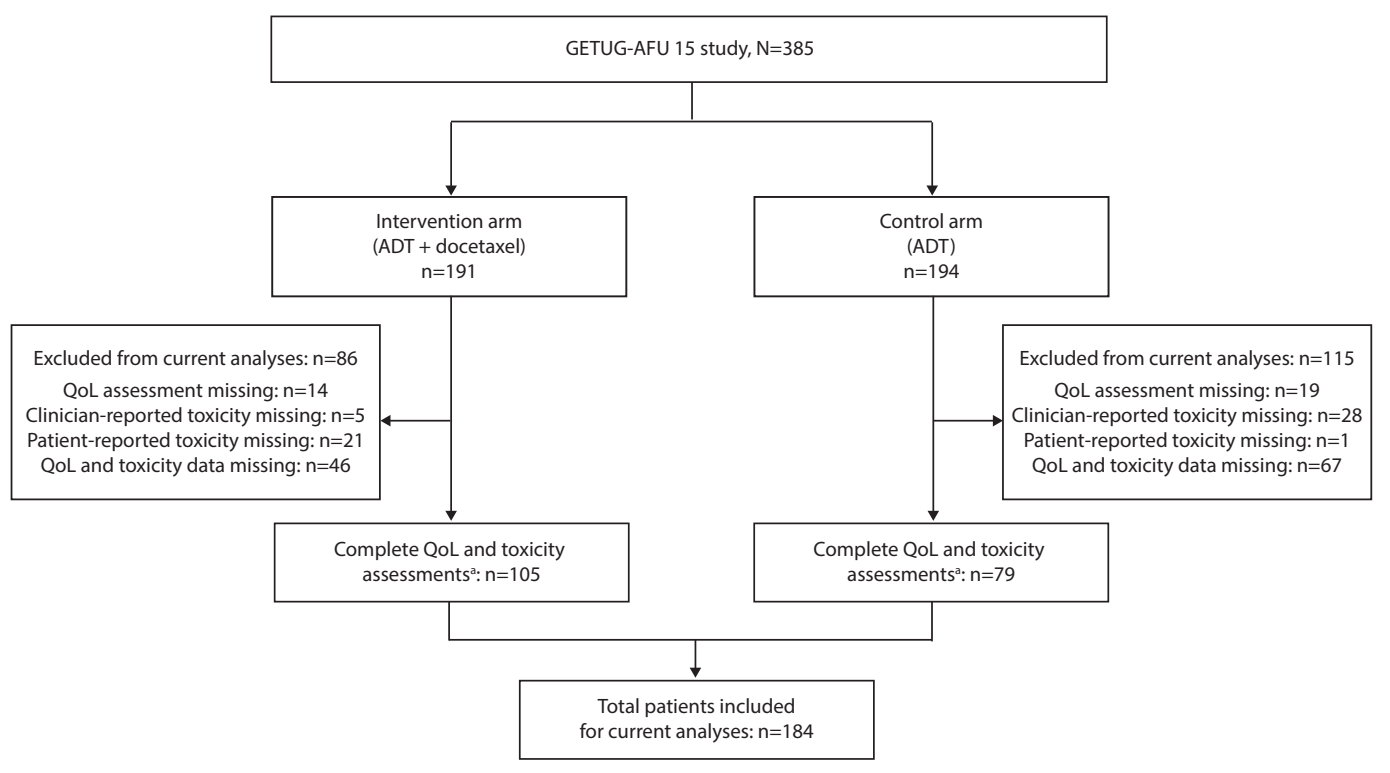

Figure 1. Study flow chart.

Abbreviations: ADT, androgen deprivation therapy; QoL, quality of life.

${ }^{a}$ Complete assessment defined as follows: QoL: baseline and $\geq 1$ follow-up measurement; clinician-reported toxicity: $\geq 1$ measurement; patient-reported toxicity: $\geq 1$ measurement.

(86.9\%) after 6 months of treatment (Table 2). As reported previously, ${ }^{3}$ the most commonly reported AEs by clinicians were hot flushes $(45 \%)$ and fatigue $(36.4 \%)$, whereas those commonly reported by patients were sexual problems, hot flushes, and fatigue (all $>70 \%$ ). Mean cumulative toxicity scores reported by patients were all significantly higher than mean scores reported by clinicians ( $P \leq .001$ for all; Table 3$)$. Cumulative toxicity assessed with all-grade AEs and severity score showed significant but poor agreement between patients and clinicians (ICCs $\leq 0.50$ ).

Association Between QoL and Cumulative Toxicity Comprising All-Grade Versus High-Grade AEs: A higher total number of all-grade $A E s(B,-2.22$; 95\% CI, -3.32 to $-1.11 ; P \leq .001)$ and a higher severity score $(\mathrm{B},-1.45 ; 95 \% \mathrm{CI},-2.18$ to $-0.71 ; P \leq .001)$ reported by clinicians were significantly associated with lower physical QoL (Table 4). The cumulative toxicity score measured by the total of only highgrade AEs did not reach the standard level of statistical significance. None of the cumulative toxicity scores were associated with global QoL (Table 4).

All patient-reported cumulative toxicity scores were significantly associated with lower global and physical QoL (all P $\leq .001$; Table 4). The standardized regression coefficients showed that both the allgrade $(\beta,-0.43 ; 95 \% \mathrm{CI},-0.5$ to -0.3$)$ and severity scores $(\beta,-0.42$; $95 \% \mathrm{CI},-0.5$ to -0.3$)$ were more as- sociated with global QoL than the high-grade score $(\beta,-0.33 ; 95 \% \mathrm{CI},-0.4$ to -0.2 ; Table 4$)$. The same pattern was observed for physical QoL: all-grade $(\beta$, $-0.45 ; 95 \% \mathrm{CI},-0.6$ to -0.3$)$ and severity scores $(\beta$, -0.48 ; $95 \% \mathrm{CI},-0.6$ to -0.4$)$ were more associated with physical QoL than the high-grade score $(\beta$, -0.36 ; $95 \%$ CI, -0.5 to -0.3 ; Table 4).

\section{Association Between QoL and Patient-Reported Versus Clinician-Reported Cumulative Toxicity:} Patient-reported cumulative toxicity was associated with global QoL, regardless of the toxicity measure (all-grade score: $\mathrm{B},-2.61 ; 95 \% \mathrm{CI},-3.31$ to -1.91 ; high-grade score: $\mathrm{B},-2.81 ; 95 \% \mathrm{CI},-3.65$ to -1.97 ; severity score: $\mathrm{B},-0.91 ; 95 \% \mathrm{CI},-1.14$ to -0.68$)$, whereas clinician-reported cumulative toxicity was not (all P>.05; Table 4).

With regard to physical QoL, the patient-reported all-grade score $(\beta,-0.45 ; 95 \% \mathrm{CI},-0.57$ to $-0.32)$ and severity score $(\beta,-0.48 ; 95 \% \mathrm{CI},-0.59$ to -0.36) showed stronger associations than clinicianreported all-grade score $(\beta,-0.24 ; 95 \% \mathrm{CI},-0.35$ to $-0.12)$ and severity score $(\beta,-0.22 ; 95 \% \mathrm{CI},-0.34$ to -0.11). For high-grade scores, the clinician-reported score was not significantly associated with physical QoL, whereas the patient-reported score was (B, $-2.39 ; 95 \% \mathrm{CI},-3.13$ to -1.66 ; Table 4$)$. 
Schuurhuizen et al

\begin{tabular}{|c|c|c|c|c|c|c|}
\hline & \multicolumn{3}{|c|}{ Reporting of AEs Between Baseline and 3 mo } & \multicolumn{3}{|c|}{ Reporting of AEs Between 3 and 6 mo } \\
\hline & $\begin{array}{l}\text { Patients Assessed for } \\
\text { Toxicity, N }\end{array}$ & $\begin{array}{c}\text { Any-Grade AEs, } \\
\mathrm{n}(\%)\end{array}$ & $\begin{array}{c}\text { High-Grade AEs, } \\
\mathrm{n}(\%)\end{array}$ & $\begin{array}{l}\text { Patients Assessed for } \\
\text { Toxicity, N }\end{array}$ & $\begin{array}{c}\text { Any-Grade AEs, } \\
\mathrm{n}(\%)\end{array}$ & $\begin{array}{c}\text { High-Grade AEs, } \\
\mathrm{n}(\%)\end{array}$ \\
\hline Clinicians & 183 & 159 (86.9) & $19(10.4)$ & 120 & $108(90)$ & $24(20)$ \\
\hline Patients & 168 & $168(100)$ & 149 (88.7) & 107 & $107(100)$ & 93 (86.9) \\
\hline
\end{tabular}

Abbreviation: $A E$, adverse event.

\section{Discussion}

The first objective of this study was to determine whether cumulative toxicity comprising all-grade AEs (including low-grade) was more associated with $\mathrm{QoL}$ than cumulative toxicity comprising high-grade AEs only. In the current analysis of patients with mCNPC, we found support for our hypothesis that cumulative toxicity scores comprising all-grade AEs reported by clinicians were associated with lower physical QoL. All patient-reported cumulative toxicity measures, including the high-grade score, were associated with lower global and physical QoL. The standardized regression coefficients demonstrated the strongest associations for the all-grade and severity scores. We also found support for our second hypothesis that all patient-reported toxicity scores were more associated with QoL outcomes than clinician-reported.

These findings indicate that the evaluation of toxicity in patients with metastatic cancer undergoing systemic treatment could be improved in 2 ways. First, if the assessment of AEs is clinician-based, cumulative toxicity scores comprising all-grade AEs (ie, including low-grade) are to be preferred over cumulative toxicity scores comprising high-grade AEs only. Second, patient-reported cumulative toxicity scores are to be preferred over clinician-based toxicity scores regarding their impact on QoL. The significance of patientreported outcomes in symptom assessment has been demonstrated previously, ${ }^{21,22}$ and recently the use of patient-reported outcomes in symptom monitoring among patients with metastatic cancer was shown to be associated with increased survival compared with usual care. ${ }^{23}$ In line with previous studies, ${ }^{24,25}$ our results demonstrated that cumulative AE scores reported by patients were more strongly correlated with (all) QoL scores than those reported by clinicians.

Our study should be interpreted in the context of several considerations. First, although patients and clinicians assessed the same symptoms, they did not complete identical questionnaires. This could partially explain the discordance between patients' and clinicians' evaluation regarding the presence and grading of AEs. In addition, symptoms that were not mentioned by clinicians were considered absent; this could have been a source of inaccuracy, because clinicians may not have asked about certain symptoms and therefore may not have included them, which does not mean they were not actually present. Second, the symptom questionnaire for patients has not been formally validated. Yet, it has been used multiple times previously and a high agreement between the scoring of identical AEs on the EORTC QLQC30 has been shown. ${ }^{3,14}$ Third, there was a substantial proportion of missing data, which in general is a major concern preventing the routine incorporation of patient-reported outcomes in RCTs. Often, substantial numbers of patients do not have complete patient-reported data due to discontinuation of treatment or disease progression, ${ }^{26}$ which is a potential confounding factor especially when evaluating toxicity. As reported previously, ${ }^{3}$ approximately half of the 385 patients included in the GETUG-AFU 15 trial completed the QoL assessments at baseline and after 3 months of treatment, which could have led to a risk of bias in our study results. Still, the combined participation rates of patient-reported AEs and QoL data are comparable with other clinical RCTs assessing QoL in patients with cancer. ${ }^{27}$ In addition, clinical character-

\begin{tabular}{|c|c|c|c|}
\hline & $\begin{array}{c}\text { Clinician-Reported Cumulative Toxicity Scores, } \\
\text { Mean (SD) }\end{array}$ & $\begin{array}{l}\text { Patient-Reported Cumulative Toxicity Scores, } \\
\text { Mean (SD) }\end{array}$ & $\begin{array}{l}\text { ICC }(95 \% \mathrm{Cl}) \\
\quad P \text { Value }\end{array}$ \\
\hline High-grade score & $0.2(0.4)$ & $3.1(2.6)$ & $0.019(-0.103 ;-0.140), P=.382$ \\
\hline All-grade score & $2.2(1.9)$ & $7.1(3.5)$ & $0.344(0.232 ;-0.447), P=.000$ \\
\hline Severity score & $3.1(2.9)$ & $16.4(10.2)$ & $0.175(0.054 ;-0.290), P=.002$ \\
\hline
\end{tabular}

Abbreviation: ICC, intraclass correlation coefficient. 
Predictive Value of Cumulative Toxicity

\begin{tabular}{|c|c|c|c|c|c|c|}
\hline & \multicolumn{3}{|c|}{ Clinician-Reported Cumulative Toxicity Scores } & \multicolumn{3}{|c|}{ Patient-Reported Cumulative Toxicity Scores } \\
\hline & B $(95 \% \mathrm{Cl})$ & $\beta(95 \% \mathrm{Cl})$ & $P$ Value & B $(95 \% \mathrm{Cl})$ & $\beta(95 \% \mathrm{Cl})$ & $P$ Value \\
\hline High-grade score ${ }^{a, b}$ & $2.615(-2.772 ; 8.001)$ & $0.048(-0.051 ; 0.147)$ & .340 & $-2.813(-3.654 ;-1.973)$ & $-0.334(-0.433 ;-0.234)$ & $<.001^{\mathrm{e}}$ \\
\hline All-grade score ${ }^{a, c}$ & $-1.215(-2.525 ; 0.095)$ & $-0.101(-0.209 ; 0.008)$ & .069 & $-2.612(-3.310 ;-1.913)$ & $-0.433(-0.547 ;-0.317)$ & $<.001^{\mathrm{e}}$ \\
\hline Severity score $\mathrm{e}^{\mathrm{a}, \mathrm{d}}$ & $-0.722(-1.589 ; 0.145)$ & $-0.087(-0.191 ; 0.017)$ & .102 & $-0.909(-1.136 ;-0.682)$ & $-0.419(-0.523 ;-0.314)$ & $<.001^{\mathrm{e}}$ \\
\hline High-grade score ${ }^{a, b}$ & $-3.710(-8.299 ; 0.879)$ & $-0.877(-0.196 ; 0.021)$ & .113 & $-2.392(-3.127 ;-1.657)$ & $-0.364(-0.476 ;-0.252)$ & $<.001^{\mathrm{e}}$ \\
\hline All-grade score ${ }^{a, c}$ & $-2.216(-3.319 ;-1.113)$ & $-0.236(-0.353 ;-0.118)$ & $<.001 \mathrm{e}$ & $-2.117(-2.711 ;-1.523)$ & $-0.450(-0.577 ;-0.324)$ & $<.001^{e}$ \\
\hline Severity score $\mathrm{s}^{\mathrm{a}, \mathrm{d}}$ & $-1.446(-2.181 ;-0.711)$ & $-0.224(-0.337 ;-0.110)$ & $<.001^{\mathrm{e}}$ & $-0.806(-1.002 ;-0.610)$ & $-0.476(-0.592 ;-0.361)$ & $<.001^{\mathrm{e}}$ \\
\hline
\end{tabular}

Abbreviations: $A E$, adverse event; $\beta$, standardized regression coefficient; $B$, unstandardized regression coefficient; QoL, quality of life.

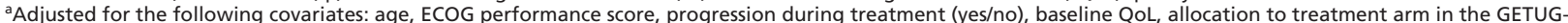

AFU 15 study, metastatic volume (high or low; the presence of visceral metastases and/or at least 4 bone lesions, including at least 1 bone structure beyond the spine or

pelvis), ${ }^{15}$ serum concentration of prostate-specific antigen at start of treatment ( $<65$ or $\geq 65 \mathrm{ng} / \mathrm{mL}$ ), and Gleason score at baseline (2-6, 7, 8-10).

${ }^{6}$ Total number of severe AEs (grade 3-4).

'Total number of AEs (all grades).

${ }^{\mathrm{d}}$ Sum of total number of all-grade AEs multiplied by their grade.

eStatistical significance was concluded at the 2-sided significance level of $\leq .05$ for these results.

istics of patients with complete QoL assessments did not significantly differ from those of patients who did not complete QoL assessments, suggesting that this study population was a representative sample of the GETUG-AFU 15 trial. Fourth, this study included patients with $\mathrm{mCNPC}$, of whom only half received chemotherapy, and the degree of reported toxicity in general was modest. ${ }^{28}$ This may affect the generalizability of the results to patients with other tumor types, treatments, and treatment toxicities. More specifically, clinicians only reported high-grade AEs in a low proportion of patients $(10.4 \%$ and $20 \%$ after 3 and 6 months, respectively). Although there was no significant association between clinician-reported high-grade cumulative toxicity scores with global and physical QoL, this could have been the result of lack of power instead of lack of effect. However, in our previous study conducted in patients with metastatic CRC, with higher rates of clinician-reported grade 3-4 AEs, a similar lack of effect of clinician-reported high-grade cumulative toxicity on QoL was observed during palliative systemic treatment. ${ }^{8}$

Future prospective studies are needed to improve toxicity and QoL evaluation, thereby addressing the limitations of our study. These should ensure the use of reliable instruments to assess toxicity, such as the CTCAE for clinicians and the recently validated PRO-CTCAE instrument for patients, ${ }^{11} \mathrm{com}$ bined with the global QoL and physical functioning subscales of the well-known EORTC-QLQ C30 or Functional Assessment of Cancer Therapy (FACT) questionnaire to assess QoL. Additionally, when assessing both toxicity and QoL in patients, one should be cautious in timing these assessments, thereby preventing the risk of priming certain overlapping questions. Furthermore, electronic collection of patient-reported data is encouraged, because this has been shown to improve compliance rate in several studies. ${ }^{29,30}$ Lastly, though we have seen that the impact of cumulative toxicity comprising all-grade AEs on QoL in patients with metastatic CRC was similar to the impact seen here in patients with mCNPC, this still must be studied in patients with other cancer stages and treatment types.

A major strength of this study is that the data obtained from clinicians and patients were collected prospectively at multiple time points. In contrast, most previous studies reporting on patient-reported (toxicity) measures used cross-sectional data. ${ }^{31,32}$ Furthermore, we included a large sample and a homogeneous group of patients that participated in a multicenter trial. Finally, we provided a solid basis for improving future reporting of toxicity by offering a new set of tools consisting of patient self-reported cumulative toxicity scores that correlate well with patients' QoL.

\section{Conclusions}

The standard methods for reporting on AEs during systemic treatment, which mainly rely on clinicianreported high-grade AEs, could be questioned. We proposed an alternative approach for handling toxicity data. Clinician-based cumulative toxicity scores comprising all-grade AEs provide a better measure of treatment burden than toxicity scores comprising high-grade AEs only. To assess the impact of toxicity on QoL, patient-based cumulative toxicity scores should be preferred. 


\section{References}

1. Friese CR, Harrison JM, Janz NK, et al. Treatment-associated toxicities reported by patients with early-stage invasive breast cancer. Cancer 2017;123:1925-1934.

2. Davis C, Naci H, Gurpinar E, et al. Availability of evidence of benefits on overall survival and quality of life of cancer drugs approved by European Medicines Agency: retrospective cohort study of drug approvals 2009-13. BMJ 2017;359:j4530.

3. Gravis G, Marino P, Joly F, et al. Patients' self-assessment versus investigators' evaluation in a phase III trial in non-castrate metastatic prostate cancer (GETUG-AFU 15). Eur J Cancer 2014;50:953-962.

4. Weeks JC, Catalano PJ, Cronin A, et al. Patients' expectations about effects of chemotherapy for advanced cancer. N Engl J Med 2012;367:1616-1625.

5. Maillet D, Blay JY, You B, et al. The reporting of adverse events in oncology phase III trials: a comparison of the current status versus the expectations of the EORTC members. Ann Oncol 2016;27:192-198

6. U.S. Department of Health and Human Services. Common Terminology Criteria for Adverse Events (CTCAE). Version 4.0. Available at: http:// www.hrc.govt.nz/sites/default/files/CTCAE\%20manual\%20-\%20DMCC. pdf. Accessed November 20, 2018.

7. Thanarajasingam G, Hubbard JM, Sloan JA, Grothey A. The imperative for a new approach to toxicity analysis in oncology clinical trials. J Natl Cancer Inst 2015;107:pii: djv216.

8. Schuurhuizen C, Konings I, Braamse A, et al. The predictive value of cumulative toxicity for quality of life in patients with metastatic colorectal cancer during first-line palliative chemotherapy. Cancer Manag Res 2018;10:3015-3021.

9. Fromme EK, Eilers KM, Mori M, et al. How accurate is clinician reporting of chemotherapy adverse effects? A comparison with patient-reported symptoms from the Quality-of-Life Questionnaire C30. J Clin Oncol 2004;22:3485-3490.

10. Di Maio M, Basch E, Bryce J, et al. Patient-reported outcomes in the evaluation of toxicity of anticancer treatments. Nat Rev Clin Oncol 2016;13:319-325.

11. Dueck AC, Mendoza TR, Mitchell SA, et al. Validity and reliability of the US National Cancer Institute's Patient-Reported Outcomes Version of the Common Terminology Criteria for Adverse Events (PRO-CTCAE). JAMA Oncol 2015;1:1051-1059.

12. Gravis G, Fizazi K, Joly F, et al. Androgen-deprivation therapy alone or with docetaxel in non-castrate metastatic prostate cancer (GETUG-AFU 15): a randomised, open-label, phase 3 trial. Lancet Oncol 2013;14:149158.

13. Aaronson NK, Ahmedzai S, Bergman B, et al. The European Organization for Research and Treatment of Cancer QLQ-C30: a quality-of-life instrument for use in international clinical trials in oncology. J Natl Cancer Inst 1993;85:365-376.

14. Macquart-Moulin G, Viens P, Bouscary ML, et al. Discordance between physicians' estimations and breast cancer patients' self-assessment of side-effects of chemotherapy: an issue for quality of care. Br J Cancer 1997;76:1640-1645.

15. Ringash J, O'Sullivan B, Bezjak A, et al. Interpreting clinically significant changes in patient-reported outcomes. Cancer 2007;110:196-202.

16. Osoba D, Rodrigues G, Myles J, et al. Interpreting the significance of changes in health-related quality-of-life scores. J Clin Oncol 1998;16:139_ 144.
17. Koo TK, Li MY. A guideline of selecting and reporting intraclass correlation coefficients for reliability research. J Chiropr Med 2016;15:155-163.

18. Twisk JW. Applied Longitudinal Data Analysis for Epidemiology: A Practical Guide, 2nd ed. Amsterdam, the Netherlands: Cambridge University Press; 2013.

19. Gravis G, Boher JM, Joly F, et al. Androgen deprivation therapy (ADT) plus docetaxel versus ADT alone in metastatic non castrate prostate cancer: impact of metastatic burden and long-term survival analysis of the randomized phase 3 GETUG-AFU15 trial. Eur Urol 2016;70:256-262.

20. Colan SD. The why and how of $Z$ scores. J Am Soc Echocardiogr 2013;26:38-40

21. Di Maio M, Gallo C, Leighl NB, et al. Symptomatic toxicities experienced during anticancer treatment: agreement between patient and physician reporting in three randomized trials. J Clin Oncol 2015;33:910-915.

22. Basch E. New frontiers in patient-reported outcomes: adverse event reporting, comparative effectiveness, and quality assessment. Annu Rev Med 2014;65:307-317.

23. Basch E, Deal AM, Dueck AC, et al. Overall survival results of a trial assessing patient-reported outcomes for symptom monitoring during routine cancer treatment. JAMA 2017;318:197-198.

24. Christodoulou M, McCloskey $P$, Stones $\mathrm{N}$, et al. Investigation of a patient reported outcome tool to assess radiotherapy-related toxicity prospectively in patients with lung cancer. Radiother Oncol 2014;112:244-249.

25. Basch E, Jia X, Heller G, et al. Adverse symptom event reporting by patients vs clinicians: relationships with clinical outcomes. J Natl Cancer Inst 2009; 101:1624-1632

26. Mercieca-Bebber R, Palmer MJ, Brundage $M$, et al. Design, implementation and reporting strategies to reduce the instance and impact of missing patient-reported outcome (PRO) data: a systematic review. BMJ Open 2016;6:e010938.

27. Ganz PA, Gotay CC. Use of patient-reported outcomes in phase III cancer treatment trials: lessons learned and future directions. J Clin Oncol 2007;25:5063-5069.

28. Marino P, Sfumato P, Joly F, et al. Q-TWiST analysis of patients with metastatic castrate naive prostate cancer treated by androgen deprivation therapy with or without docetaxel in the randomised phase III GETUGAFU 15 trial. Eur J Cancer 2017;84:27-33.

29. Movsas B, Hunt D, Watkins-Bruner D, et al. Can electronic web-based technology improve quality of life data collection? Analysis of Radiation Therapy Oncology Group 0828. Pract Radiat Oncol 2014;4:187-191.

30. Basch E, Pugh SL, Dueck AC, et al. Feasibility of patient reporting of symptomatic adverse events via the Patient-Reported Outcomes Version of the Common Terminology Criteria for Adverse Events (PRO-CTCAE) in a chemoradiotherapy cooperative group multicenter clinical trial. Int Radiat Oncol Biol Phys 2017;98:409-418.

31. Barata A, Martino R, Gich I, et al. Do patients and physicians agree when they assess quality of life? Biol Blood Marrow Transplant 2017;23:10051010.

32. Jensen K, Bonde Jensen A, Grau C. The relationship between observerbased toxicity scoring and patient assessed symptom severity after treatment for head and neck cancer. A correlative cross sectional study of the DAHANCA toxicity scoring system and the EORTC quality of life questionnaires. Radiother Oncol 2006;78:298-305. 